\title{
CLINICAL AND RADIOGRAPHIC EVALUATION OF MAXILLARY SMALL DIAMETER IMPLANT SUPPORTED SCREW RETAINED RESTORATIONS: A TWENTY-FOUR MONTHS FOLLOW-UP
}

\author{
Heba E. Khorshid* and Nora M. Sheta*
}

\begin{abstract}
Purpose: The Target of this work was to clinically and radiographically evaluate the marginal bone loss and stability of Small Diameter Implants in comparison with Standard Diameter implants in implant supported screw retained maxillary prostheses.

Materials and Methods: In this study, a total of seventy two implants were placed in twelve patients with completely edentulous maxillae. The patients were randomly grouped into two groups: Group A received small diameter implant and Group B received standard diameter implants. For each patient, six implants were placed in the lateral incisor/Canine region, first premolar and first molar region. Bone height measurements as well as Osstell RFA (resonance frequency analysis) measurements around each implant were performed at zero, four, eight and twenty-four months after definitive prostheses delivery.
\end{abstract}

Results: In this study, a total of 72 implants were installed (36 small diameter implants and 36 standard diameter implants). At the end of the 24 months follow-up period, statistical analysis showed no statistically significant difference in the crestal bone height and ISQ (implant stability quotient) values $(\mathrm{p} \leq 0.05)$ between the Group A and Group B.

Conclusion: Within the limited observation period and the number of patients included in this study, it may be concluded that the use of small-diameter implants appeared to be predictable if proper clinical guidelines are followed and appropriate prosthetic restorations are provided. Small-diameter implant supported screw retained restorations have success rate that appear to be comparable to that of standard diameter implants. This might thus be an efficient, low-cost solution, avoiding extensive bone augmentation procedures and reducing the surgical complexity of implant rehabilitations.

KEYWORDS: edentulous maxillae, small diameter implants, screw retained restorations.

* Lecturer, Department of Prosthodontics, Faculty of Oral and Dental Medicine, Cairo University. 


\section{INTRODUCTION}

Dental implant diameters less than the standard diameter are classified by Kelien et al ${ }^{(1)}$ according to their design into one piece or two piece and according to their diameter into narrow-diameter implants from (3.0 to3.5 mm), smaller implants (3.0 to $3.25 \mathrm{~mm})$, and mini-implants $(<3.0 \mathrm{~mm})$. The mini- implants are divided in hybrid implants (2.7 to $2.9 \mathrm{~mm}$ ) and mini implants (1.8 to $2.7 \mathrm{~mm}$ ).

Small diameter implants have been successfully used to support removable and fixed oral prostheses $^{(2,3)}$. A small diameter implant presents less of an obstacle for angiogenesis and there is less percutaneous exposure and bone displacement as compared with standard sized implants if clinical guidelines are followed and appropriate prosthetic restorations are provided. ${ }^{(4,5)}$

However, Narrow diameter implants may be at increased risk of overload thus Implants with higher fatigue strength may be beneficial. In a study performed by Al-Nawas et al. ${ }^{(6)}$ results showed that the survival and success rates were $97.8 \%$ and $97.6 \%$ respectively after 1 year; and $97.6 \%$ and $97.4 \%$ respectively after 2 years using narrow diameter (Ø $3.3 \mathrm{~mm}$ ) TiZr alloy implants for 2 years. Another study performed by Polack and Arzadon (7) presented high success rates of the immediate loading of a novel small-diameter titanium-zirconium implant, within a 6-month follow up. While in another study, the survival rate of the small-diameter and mini dental implants over 5 years reached $98.3-99.4 \% .{ }^{(8)}$ Less surgical time, less postoperative pain, ability of direct loading after surgery with no harm to bone and cost effectiveness are the advantages of these implants. ${ }^{(9)}$

The successful use of these small-diameter implants for temporary stabilization of prostheses has led many clinicians to explore the option of using them as a definitive alternative, especially as the technique requires minimal time and also is economical for the patients. Non-smokers had an implant survival of $100 \% .{ }^{(10)}$ Small-diameter implant-retained overdentures have success rates that appear to be comparable to that of regular diameter implants. They might also be an efficient, low-cost solution for elders who wish to reduce problems with denture instability. ${ }^{(11,12)}$

However, a meta-analysis performed by OrtegaOller et al. ${ }^{(13)}$ showed that narrower implants $(<3.3 \mathrm{~mm})$ had significantly lower survival rates compared with wider implants $(\geq 3.3 \mathrm{~mm})$. While another systematic review performed by Bidra and Almas ${ }^{(14)}$ reported that there is no evidence for the long-term survival of mini implants. As yet, no detailed scientific analysis of the mechanical and biomechanical impact of the reduced diameter and length of these implants has been published ${ }^{(15)}$ Brian. J ${ }^{(16)}$ summarized the cause of small implant diameter failure maybe due to poor surgical placement, immediate loading and poor occlusal design.

\section{MATERIALS AND METHODS}

Twelve male patients were selected from the outpatient clinic of the Prosthodontics Department, Faculty of Oral and Dental Medicine, Cairo University. Patients were with Completely Edentulous Maxillae showing normal maxillo-mandibular relationship (Class I Angle classification), with no para-functional habits and systemically free from any medical conditions.

In this study, patients were divided into two equal groups: Group A: Patients received implants with a 3.0mm diameter while Group B: Patients received implants with a $3.7 \mathrm{~mm}$ diameter.

The pre-surgical preparation required the construction of conventional maxillary complete dentures. The finished maxillary dentures were duplicated to obtain radio-opaque scan appliances. Duplication was performed using a mixture of amalgam powder and transparent self-cured acrylic resin powder. The patients' maxillae 
were radiographed using Cone Beam Computed Tomographic (CBCT) scanning machine (Sanora 3D Soredex, Helsinki, Finland). During imaging the patients were instructed to wear their stents and to stabilize it in place by biting on an occlusal index constructed for each patient, separating the mandibular teeth from the stent. DICOM files obtained from the CT scan were loaded into the Mimics software (Mimics, Materialise HQ, Technologielaan 15, 3001 Leuven, Belgium) whereby coronal and sagittal reformatting and panoramic views were obtained. The desired implant sites were identified through the radiolucent channels previously prepared in the radiographic stent at the prosthetic teeth centers. The bone volumes at each of the six potential sites were evaluated for sufficient bone height, width and density. For each patient, six implants were to be planned in the lateral incisor/ Canine region, first premolar and first molar region according to the available bone height and width. All Implants were with standardized height; $13 \mathrm{~mm}$ for the four anterior implants and $10 \mathrm{~mm}$ for the two posterior implants. The virtual STL files of the implants were imported into the MIMICS software and then virtual planning was performed at the proposed implant sites (Figure 1).

The base of the radiographic stent was separated from the bone and teeth using the segmentation

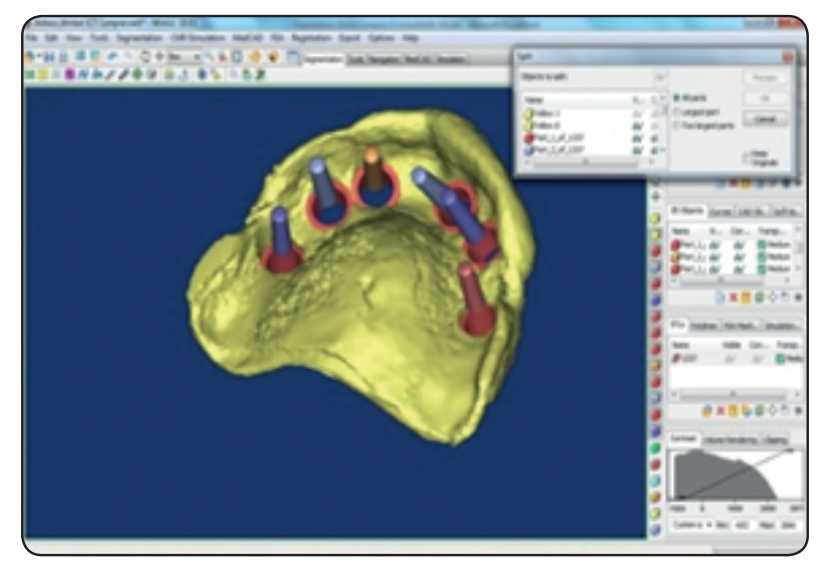

Fig. (1) "Split" Operation being performed process. The created mask of the base was grown to a 3D object and then united with the supra bony portion of the implant model using the "Boolean operation" tool. The resultant object is the $3 \mathrm{D}$ virtual stent which was exported as an STL (Sterolithiographic) file for 3D printing machine (Invision Si2, USA) to build the stent from a photo curable resin material. Metallic sleeves were fitted into the designed holes of the fabricated stent and then the stent was tried in the patient's mouth to check stability and fit.

\section{Implant Installation}

Before starting the surgical procedure, the perioral region of each patient was wiped by Betadine antiseptic solution, the surgical instruments were autoclaved and the computer guided stent was disinfected with a suitable disinfectant. At the time of surgery, infiltration anaesthesia was injected at each implant site. The stent was fixed in place using three fixation screws. Osteotomies were then prepared using the classical drilling sequence (pilot, intermediate and final drills) and were irrigated with sterile saline after each drill. For every drill a specially designed "drill guide" was used .The implants were then unpacked and inserted manually through the stent till manual tightening met resistance and further tightening was completed with a ratchet using a depth controlling implant driver (Figure 2).

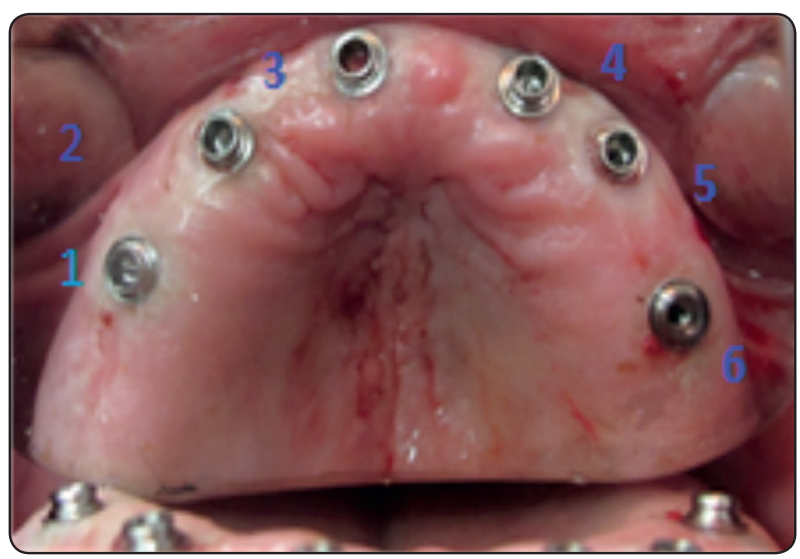

Fig. (2) Implants after being surgically installed and stent Retrieval 
The primary stability of each implant was checked using "Osstell"* ISQ device.

After 4-6 months, the patients were recalled and the Implants were checked for adequate osseointegration using "Osstell” ISQ device. The snap-on Implant plastic transfer copings supplied with the implants were placed over each implant and preliminary impression were then taken using a closed tray technique with medium body rubber base impression material. The implant analogues** were then snapped on over the Plastic transfer copings inside the impression and then the impression was poured using medium hard stone.

Temporary Titanium abutments were then screwed over the implant analogues within the primary cast and then splinted together using DuraLay resin material***. The framework was then tried in the patient's mouth and screwed over the implants. The passive fit was checked using the one screw test and using an intraoral explorer. If any areas were detected with lack of passivity, sectioning of the duralay splint was performed and then re-connected intraorally again using Duralay. After complete set of the Duralay, passive fit was then checked finally. The radiographic stents were then modified by opening windows at areas of the implants and used as a special tray. An open tray impression technique was then performed and again the implant analogues were screwed over the temporary titanium abutments. After pouring of the master cast, plastic castable abutments ${ }^{* * * *}$ were fastened to the analogues. The plastic abutments were connected with Duralay resin to form a rigid frame. The pattern was invested and cast into chrome cobalt alloy.

The frameworks for both groups were checked individually for fit and passivity using the one screw test was performed. The detection of any gap is an indication that sectioning with a disc, and fastening separately to the implants, re-connecting with Duralay resin and soldering (or welding) was required.

Bite registration was then performed using the Wax wafer registration method. Acrylic teeth were set on the framework following the IPO guidelines in accordance with Misch's recommendations. Visio-lign Veneering (Visio-lign, Bredent $\mathrm{GmbH}$ \& Co.KG, WeissenhornerSenden, Germany) light cured system was used to construct the gingiva using a free-hand technique.

After the build-up is complete, the screw-retained implant supported prostheses were screwed intraorally and fine occlusal adjustments were made in both groups (Figure $3 \boldsymbol{A}, \boldsymbol{B}$ ). The prosthetic screws were tightened to $30 \mathrm{Ncm}$ with a torque wrench. The access holes were partially plugged with rubber pieces and completely blocked with light-cured composite resin restorative material.

In this study, Implants were nominated from 1 to 6 starting from the right hand side to the left hand side of each patient. Each patient performed three follow-up CT scans using CBCT machine.*****. The CT scans were performed at zero, four, eight and twenty four months after definitive prostheses delivery. The raw DICOM data obtained from the CBCT scanning were imported to special third party software $^{* * * * * * *}$ for secondary reconstruction. Results obtained from the data sets were compared to each other (Figure 4). The Osstell was used to measure the implant stability at zero, four, eight and twenty four months after definitive prostheses delivery. The numbers obtained were then tabulated and statistically analyzed.

\footnotetext{
* Osstell AB, Gamlestadsvägen 3B, SE415 02, Sweden.

** ImplantDirectTM LLC Spectra-System Dental Implants) Calabasas Hills CA, USA

*** DuraLayTM, Reliance, Dental MFG Co. Worth, IL, USA

**** Plastic burnoutsImplants, ImplantDirectTM LLC Spectra-System Dental Implants Calabasas Hills CA, USA

***** Scanora 3D Soredex, Helsinki, Finland

****** Ondemand 3D, Seoul, South Korea
} 


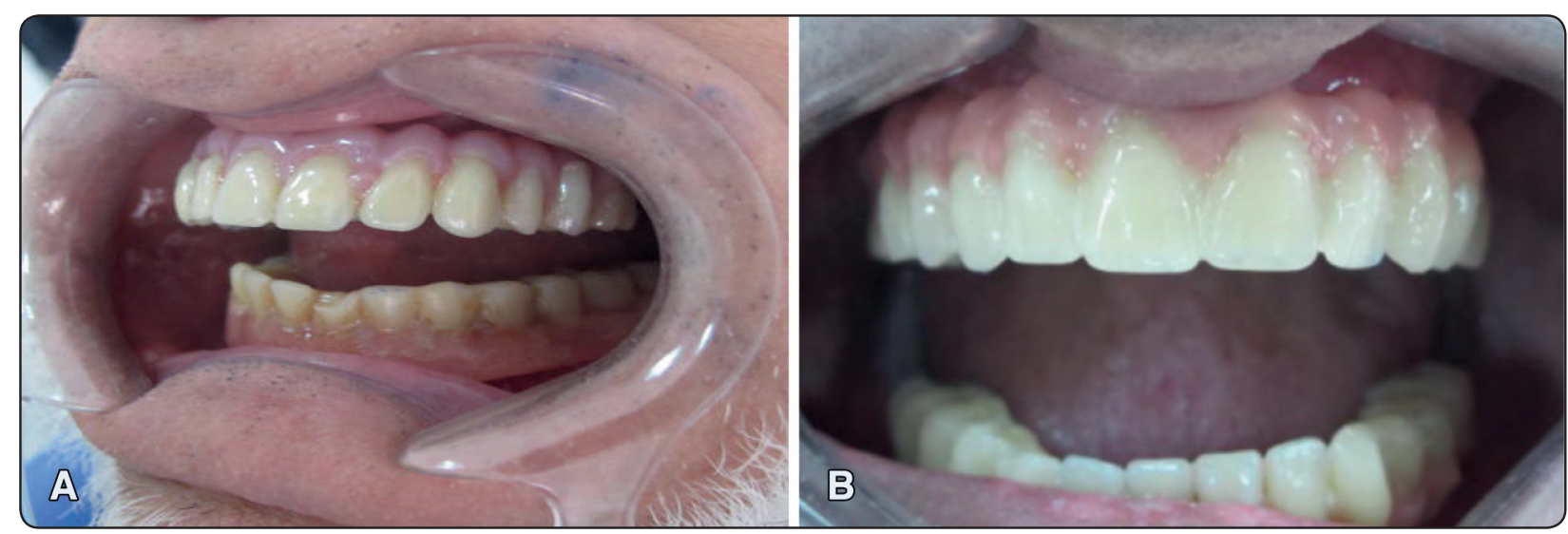

Fig. (3) (A) The screw-retained implant supported prostheses delivered in the patient's mouth for GROUPA (B) The screw-retained implant supported prostheses delivered in the patient's mouth for GROUP B

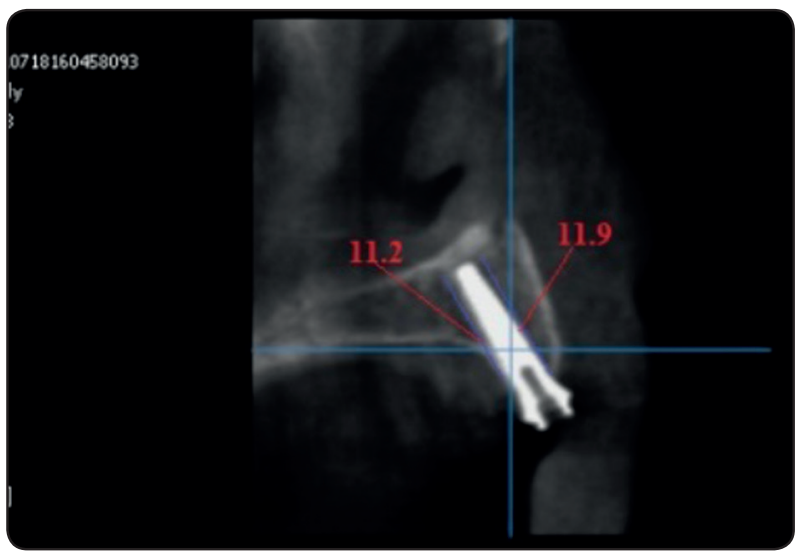

Fig. (4) Buccal and palatal Bone Height measurements

\section{Statistical Analyses}

The results of this study were statistically analysed to evaluate the changes that occurred in the supporting structures of each implant placed in the maxilla as a result of the use of two different implant diameters. Implants were nominated from 1 to 6 starting from the right hand side to the left hand side of each patient. The implant bone height measurements and the resonance frequency analysis measurements (Implant Stability Quotient ISQ) surrounding each implant were used to evaluate the hard tissue reactions in both groups at zero, four, eight and twenty four months after definitive prostheses delivery.

\section{Statistical Methods}

The normal distribution of parameters was tested by the Shapiro-Wilk test. Since the data was non-parametric, Mann-Whitney $U$ and Wilcoxon rank sum tests were utilized. All the measurements in the text and Tables were described as median and interquartile range (IQR). Probability values $(p \leq 0.05)$ were considered statistically significant. All calculations were made using the SPSS Statistics software Version 13.0: SPSS Inc.

\section{RESULTS}

\section{Bone Height}

In this study, The median and the interquartile range (IQR) of the bucco-palatal bone height in Imp1, Imp2, Imp3, Imp4, Imp5 and Imp6 were $-0.68(0.92) \mathrm{mm},-0.73(1.31) ' \mathrm{~mm},-1.03(1.14) \mathrm{mm}$, $-1.38(2.39) \mathrm{mm},-0.73(1.88) \mathrm{mm}$ and $-1.83(2.26)$ $\mathrm{mm}$ in Group A from zero to twenty four months of prostheses delivery respectively. The median and the interquartile range (IQR) of the bucco-palatal bone height in Imp1, Imp2, Imp3, Imp4, Imp5 and Imp6 were $-0.68(1.13) \mathrm{mm},-1.18(1.43) \mathrm{mm}$, $-1.30(1.40) \mathrm{mm},-1.23(3.01) \mathrm{mm},-1.13(1.84) \mathrm{mm}$ and $-0.73(1.53) \mathrm{mm}$ in Group B from zero to twenty four months of prostheses delivery respectively. Throughout the whole study period; the Mann- 
Whitney U test showed no statistically significant difference between the Bucco-palatal bone height measurements in the two groups $(\mathrm{p} \leq 0.05)$.

The median and the interquartile range (IQR) of the mesio-distal bone height in Imp1, Imp2, Imp3, Imp4, Imp5 and Imp6 were -0.65(1.25) $\mathrm{mm},-1.20(0.89) \mathrm{mm},-1.13(1.08) \mathrm{mm},-1.05$ (0.95)mm, $-1.25(1.16) \mathrm{mm}$ and $-1.60(0.86) \mathrm{mm}$ in Group A from zero to twenty four months of prostheses delivery respectively. The median and the interquartile range (IQR) of the mesio-distal bone height in Imp1, Imp2, Imp3, Imp4, Imp 5 and Imp 6 were $-1.15(1.00) \mathrm{mm}-1.30(1.60) \mathrm{mm}$, $-0.97(2.63) \mathrm{mm},-1.48(2.36) \mathrm{mm},-1.18 \quad(1.85)$ $\mathrm{mm}$ and $-0.65(1.31) \mathrm{mm}$ in Group B from zero to twenty four months of prostheses delivery respectively. Throughout the whole study period; the Man n-Whitney $U$ test showed no statistically significant difference between the mesio-distal bone height measurements in the two groups $(\mathrm{p} \leq 0.05)$ as shown in Table 1.
In this study, Statistical analysis revealed no statistically significant difference in the Bone height measurements in the time periods $\mathrm{T} 1, \mathrm{~T} 2$ and T3 between Group A and Group B throughout the whole study period. Statistical analysis also showed that the bone height changes by time within group A were statistically significant. The median and the interquartile range (IQR) of the bone height at $\mathrm{T} 1$ (zero to four), T2 (four to eight), T3 (eight to twenty four) months were $10.5(1.6) \mathrm{mm}, 10.2(2.6) \mathrm{mm}$ and 10.2 (2.5) mm respectively using Wilcoxon rank sum tests. As reported in Table 2, differences at a statistically-significant level were recorded between T1, T2 and T3 P $(<0.001)$. Statistical analysis also showed that the bone height changes by time within Group B were statistically significant. The median and the interquartile range (IQR) of bone height at T1, T2, T3 were 10.8 (1.9) mm, 10.4 (1.7) $\mathrm{mm}$ and $10.35(1.8) \mathrm{mm}$ respectively using Wilcoxon rank sum tests. As reported in Table 2, differences at a statistically-significant level were recorded between $\mathrm{T} 1, \mathrm{~T} 2$ and $\mathrm{T} 3 \mathrm{P}(<0.001)$.

TABLE (1) The Median differences, Interquartile Range and P values for comparing the Bucco-lingual and Mesio-distal bone heights between the two groups from 0 to 24 months follow up period

\begin{tabular}{|c|c|c|c|c|c|}
\hline $\begin{array}{c}\text { Time } \\
\text { From 0 to 24 month }\end{array}$ & \multicolumn{2}{|c|}{$\begin{array}{c}\text { Group A } \\
\text { Small implant diameter }\end{array}$} & Standard implant diameter & IQR & P value \\
\hline $\begin{array}{c}\text { Implant number } \\
\text { Bucco-palatal(BP) and Mesio-distal(MD) }\end{array}$ & Median & IQR & Median & & 0.38 \\
\hline Imp1 BP & -0.68 & 0.92 & -0.68 & 1.13 & 0.57 \\
\hline Imp1 MD & -0.65 & 1.25 & -1.15 & 1.00 & 0.38 \\
\hline Imp2 BP & -0.73 & 1.31 & -1.18 & 1.43 & 1.00 \\
\hline Imp2 MD & -1.20 & 0.89 & -1.30 & 1.60 & 0.23 \\
\hline Imp3 BP & -1.03 & 1.14 & -1.30 & 1.40 & 0.75 \\
\hline Imp3 MD & -1.13 & 1.08 & -0.97 & 2.63 & 0.87 \\
\hline Imp4 BP & -1.38 & 2.39 & -1.23 & 3.01 & 1.00 \\
\hline Imp 4 MD & -1.05 & 0.95 & -1.48 & 2.36 & 0.57 \\
\hline Imp5 BP & -0.73 & 1.88 & -1.13 & 1.84 & 0.75 \\
\hline Imp 5 MD & -1.25 & 1.16 & -1.18 & 1.85 & 0.20 \\
\hline Imp6 BP & -1.83 & 2.26 & -0.73 & 1.53 & 0.33 \\
\hline Imp6 MD & -1.60 & 0.86 & -0.65 & 1.85 & \\
\hline
\end{tabular}


TABLE (2) The Median differences, Interquartile range and P values for comparing the bone height changes by time within each group at zero, four, eight and twenty four month using Wilcoxon rank sum tests.

\begin{tabular}{|c|c|c|c|c|c|}
\hline \multirow{2}{*}{$\begin{array}{c}\text { Groups } \\
\text { Group A } \\
\begin{array}{c}\text { Small Diameter } \\
\text { Implant }\end{array}\end{array}$} & Median (IQR) & $11.5(2.3)$ & $\begin{array}{c}\text { 4th month } \\
10.5(1.6)\end{array}$ & $\begin{array}{c}\text { 8th month } \\
10.2(2.6)\end{array}$ & $\begin{array}{c}24 \text { th month } \\
10.2(2.5)\end{array}$ \\
\cline { 2 - 6 } & $\mathrm{p}$ Value & & $\mathrm{T} 1(<0.001)$ & $\mathrm{T} 2(<0.001)$ & $\mathrm{T} 3(<0.001)$ \\
\hline $\begin{array}{c}\text { Group B } \\
\text { Standard Diameter } \\
\text { Implants }\end{array}$ & Median (IQR) & $11.5(2.2)$ & $10.8(1.9)$ & $10.4(1.7)$ & $10.35(1.8)$ \\
\cline { 2 - 6 } & $\mathrm{p}$ Value & $\mathrm{T} 1(<0.001)$ & $\mathrm{T} 2(<0.001)$ & $\mathrm{T} 3(<0.001)$ \\
\hline
\end{tabular}

TABLE (3) The Median differences, Interquartile range and $\mathrm{P}$ values for comparing the Bucco-palatal and mesio-distal ISQ values between the two groups from 0 to 24 months follow up period.

\begin{tabular}{|c|c|c|c|c|c|}
\hline \multirow{2}{*}{$\begin{array}{c}\text { Time } \\
\text { From 0 to 24 month }\end{array}$} & \multicolumn{2}{|c|}{$\begin{array}{c}\text { Group A } \\
\text { Small implant diameter }\end{array}$} & \multicolumn{2}{c|}{ Standard implant diameter } & IQR \\
\hline $\begin{array}{c}\text { Implant number Buccopalatal (BP) } \\
\text { and mesiodistal (MD) }\end{array}$ & Median & IQR & Median & Palue \\
\hline Imp1 BP & 3.25 & 14.13 & -1.25 & 15.00 & 0.42 \\
\hline Imp1MD & 1.50 & 8.75 & -1.00 & 6.38 & 0.57 \\
\hline Imp2 BP & -0.25 & 8.38 & -2.00 & 12.00 & 0.94 \\
\hline Imp2 MD & -2.25 & 9.25 & 0.00 & 11.50 & 0.75 \\
\hline Imp3 BP & -4.50 & 12.25 & -3.25 & 8.13 & 0.94 \\
\hline Imp3 MD & -0.75 & 12.63 & -0.50 & 10.38 & 0.75 \\
\hline Imp4 BP & -1.50 & 5.00 & -1.75 & 6.63 & 0.63 \\
\hline Imp 4 MD & -1.00 & 6.75 & -1.25 & 9.50 & 0.63 \\
\hline Imp5 BP & 0.25 & 14.25 & 1.00 & 7.25 & 0.87 \\
\hline Imp5 MD & -1.00 & 11.88 & 0.00 & 11.25 & 0.57 \\
\hline Imp6 BP & -1.25 & 2.88 & -0.50 & 4.50 & 0.47 \\
\hline Imp6 MD & -0.25 & 11.13 & 2.00 & 7.25 & 0.69 \\
\hline
\end{tabular}

\section{Osstell Results (ISQ Values)}

In this study, The median and the interquartile range (IQR) of the bucco-palatal ISQ values in Imp1, Imp2, Imp3, Imp4, Imp5 and Imp6 were 3.25 (14.13), -0.25 (8.38), -4.50 (12.25), -1.50(5.00), 0.25 (14.25) and-1.25 (2.88) ISQ in Group A from zero to twenty four months of prostheses delivery respectively. The median and the interquartile range (IQR) of the bucco-palatal ISQ values in Imp1, Imp2, Imp3, Imp4, Imp5 and Imp6 were -1.25
$(15.00), \quad-2.00(12.00), \quad-3.25(8.13),-1.75(6.63)$, $1.00(7.25)$ and 0.50 (4.50) in Group B from zero to twenty four months of prostheses delivery respectively. Throughout the whole study period; the Mann-Whitney U test showed no statistically significant difference between the bucco-palatal ISQ measurements in the two groups $(\mathrm{p} \leq 0.05)$.

The median and the interquartile range (IQR) of the mesio-distal ISQ values in Imp1, Imp2, Imp3, Imp4, Imp5 and Imp6 were 1.50(8.75), 
$-2.25(9.25),-0.75(12.63),-1.00(6.75)-1.00(11.88)$ and $-0.25(11.13)$ ISQ in Group A from zero to twenty four months of prostheses delivery respectively. The median and the interquartile range (IQR) of the mesio-distal ISQ values in Imp 1, Imp2, Imp3, Imp4, Imp5 and Imp6 were $-1.00(6.38), 0.0011 .50),-0.50(10.38),-1.25$ (9.50), 0.00 (11.25), and 2.00(7.25) in Group B from zero to twenty four months of prostheses delivery respectively. Throughout the whole study period; the Mann-Whitney U test showed no statistically significant difference between the mesio-distal ISQ measurements in the two groups $(\mathrm{p} \leq 0.05)$.

In this study, Statistical analysis revealed no statistically significant difference in the ISQ values in the time periods T1, T2 and T3 between Group A and Group B throughout the whole study period. The ISQ values change by time within group A showed no statistically significance differences. The median and the interquartile range (IQR) of the ISQ values at T1 (zero to four), T2 (four to eight), T3 (eight to twenty four) months were 58.75 (8) 58 (7.9) and 58.25 (7.5) ISQ respectively using Wilcoxon rank sum tests. The data showed no statistically significance differences in the ISQ values change by time within group B at $\mathrm{T} 1$ and $\mathrm{T} 2$. The median and the interquartile range (IQR) of ISQ at T1, T2 and T3 were 58 (5.75), 56.75 (6.9) and 57 (7) ISQ values respectively using Wilcoxon rank sum tests.

\section{DISCUSSION}

Generally, the patients who participated in this study were satisfied with their implant-supported restorations. The restorations were highly accepted by the patients due to the restoration being transformed from a removable complete denture to a fixed screw retained implant supported restoration, better masticatory function, increased comfort and elimination of the flanges. They were all able to accommodate efficiently to their restorations immediately after the prostheses delivery.

Bone Height: Statistical Analysis of the Bone height measurements in both Groups showed no statistical significant difference between the implants regarding their position in the maxillary arch and their lengths. Analysis of the median values of the peri-implant bone height revealed a reduction in the bone height around implants in both groups. At base line, there was no statistically significant difference between the bone height measurements in the two groups. This might be attributed to the fact that the interface between the implants and bone begins to remodel due to two main causes as reported by Parfitt ${ }^{(17)}$; the surgical trauma and the mechanical loading response produced by the delivery of the implant prosthesis leading to the inevitable crestal bone resorption.

TABLE (4) The median differences, Interquartile range and P values for comparing The resonance frequency analysis ISQ change by time within each group for follow-up time intervals: T1, T2 and T3 (8th to using Wilcoxon rank sum tests at zero ,four ,eight and twenty four month using Wilcoxon rank sum tests

\begin{tabular}{|c|c|c|c|c|c|}
\hline \multicolumn{2}{|c|}{ Groups } & 0 month & 4 months & 8 months & 24 months \\
Group A & Median (IQR) & $61(8.9)$ & $58.75(8)$ & $58(7.9)$ & $58.25(7.5)$ \\
Small Diameter Implant & p Value & & $\mathrm{T} 1(0.24)$ & $\mathrm{T} 2(\mathbf{0 . 0 3})$ & $\mathrm{T} 3(0.05)$ \\
Group B & Median (IQR) & $58.5(7)$ & $58(5.75)$ & $56.75(6.9)$ & $57(7)$ \\
Standard Diameter Implants & p Value & & $\mathrm{T} 1(0.08)$ & $\mathrm{T} 2(0.07)$ & $\mathbf{T 3}(<\mathbf{0 . 0 0 1})$
\end{tabular}


Statistical analysis showed no statistically significant in the crestal bone height between the Group A and Group B from zero to twenty four month. This was in accordance with multiple studies performed by Flanagan (2) and Jackson ${ }^{\text {(3) }}$ who reported that small diameter implants have been successfully used to support removable and fixed oral prostheses. According to a study performed by Al-Nawas et al. ${ }^{(6)}$ results showed that survival and success rates were $97.8 \%$ and $97.6 \%$ respectively after 1 year; and $97.6 \%$ and $97.4 \%$ respectively after 2 years using narrow diameter (Ø $3.3 \mathrm{~mm}$ ) TiZr alloy implants for 2 years. These results are also agreeable with another two studies performed by Polack and Arzadon ${ }^{(7)}$ and Hasan et al. ${ }^{(8)}$ who presented high success rates of the smalldiameter and mini dental implants over a period 6-month and 5 years respectively. Brian.J ${ }^{(16)}$ also added that the use of Small-diameter implants can be a possible solution for patients with deficient bone, compromised health histories and financial limitations. He concluded that the use of the small diameter implant must follow strict protocols to ensure predictable outcomes.

The results also demonstrated that there was a statistically significant decrease in the bone height in the time period from zero to four months and from four to eight than the time period of eight to twenty four months within each group. Roberts et al. ${ }^{(18)}$ explained that the surgical process of the implant osteotomy preparation, implant insertion and prosthetic loading cause a regional accelerated phenomenon (RAP) of bone around the implant/ bone interface resulting in crestal bone loss during the first 3 to 4 months. Enlow ${ }^{(19)}$ added that bone remodeling from mechanical strain does not only repair damaged bone but also allows the implant interface to adapt to its biomechanical situation thus explaining the reason why the bone height reduction decreased in the study period of 8 to 24 months. This was agreed upon by Slaets et al. ${ }^{(20)}$ who also demonstrated that the bone offered good adaptation possibilities rendering adequate osseointegration of immediately loaded implants.

Regarding the Resonance Frequency Analysis records obtained by the Osstell device, there was no statistically significant difference between implant stability quotient value (ISQ) values in the two groups throughout the whole study period. Statistical Analysis of the ISQ values in both Groups also revealed no statistical significant difference between the implants regarding their position in the maxillary arch and their lengths. It is hypothesized by Meredith ${ }^{(21)}$ and Friberg et al. (22) that determination of a defined implant stability quotient value (ISQ) might be relevant to predict the osseointegration prognosis of a given implant. The Resonance frequency analysis is related to the stiffness of the implant in the surrounding bony tissues as reported by Gedrange et al. ${ }^{(23)}$. In viable bone stiffness depends on the time of healing, because bone forms and remodels towards the implant surfaces as a result of the osseointegration healing process as explained by Abrahmsson et al. (24)

These results obtained were in accordance with two studies performed by Lachmann et al. ${ }^{(25)}$ and Turkyilmaz et al. ${ }^{(26)}$ where on correlating implant stability values to marginal bone level, it was found that the osstell device can detect marginal bone loss of greater to or equal to $2 \mathrm{~mm}$. As explained by Lachmann et al. ${ }^{(25)}$ and Turkyilmaz et al. ${ }^{(26)}$, the Osstell device was not able to record differences in the ISQ values in the time period of 0 and 4 months but was able to record a statistically significant difference in the time period of 4 to 8 months because the amount of bone resorption was not sufficient to be detected by the Osstell device during 0 and 4 months study periods but was sufficient eight months after restoration delivery

Statistical analysis of the ISQ values in this study within both groups revealed no statistically significant difference throughout the whole study 
period. However, there was still a numerical decrease at T1, T2 and T3 of both groups except for T3 in group B where there was actually a statistically significant increase in the ISQ values. This was agreed upon by Turkyilmaz et al. (26) and Quesada- Garcia ${ }^{(27)}$ who monitored stability changes of oxidized surface implants over a period of 12 months and found that the implant stability quotient values decreased during the first 3 month follow-up period and then increased from the third to the 12 month period. Lachmann et al. ${ }^{(25)}$ and Turkyilmaz et al. ${ }^{(26)}$ reported, where on correlating implant stability values to marginal bone level, they found that the osstell device can detect marginal bone losses of greater to or equal to $2 \mathrm{~mm}$.

\section{CONCLUSION}

Within the limited observation period and the number of patients included in this study, it may be concluded that the use of small-diameter implants appears to be predictable if proper clinical guidelines are followed and appropriate prosthetic restorations are provided. Small-diameter implant supported screw retained restorations have success rate that appear to be comparable to that of standard diameter implants. This might thus be an efficient, low-cost solution, avoiding extensive bone augmentation procedures and reducing the surgical complexity of implant rehabilitations.

\section{ACKNOWLEDGEMENT}

The authors would like to convey their special thanks to Professor Dr. Gerald A. Niznick for his invaluable help and support and for supplying the dental implants used in this study (ImplantDirect

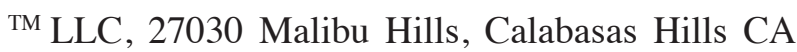
91301, USA).

\section{DISCLOSURE}

The authors claim to have no financial interest in any company or any of the products mentioned in this article.

\section{REFERENCES}

1. Klein MO, Schiegnitz E, Al-Nawas B. Systematic review on success of narrow-diameter dental implants. The International Journal of Oral \& Maxillofacial Implants 2014; 29 Supplement 43-54.

2. Flanagan D. Mini Implants Supporting Fixed Partial Dentures In The Posterior mandible: A Retrospective. J Oral Implantol. 2014 Apr 29

3. Jackson BJ. Small-diameter implants: a treatment consideration for the maxillary edentulous patient. Dent Today. 2013 Nov;32(11):86, 88, 90-2; quiz 93

4. Flanagan D. Fixed partial dentures and crowns supported by very small diameter dental implants in compromised sites. Implant Dent. 2008 Jun;17 (2):182-91.

5. Yaltirik M, Gökçen-Röhlig B, Ozer S, Evlioglu G. Clinical evaluation of small diameter straumann implants in partially edentulous patients: a 5-year retrospective study. J Dent (Tehran). 2011 Spring;8(2):75-80.

6. Al-Nawas B, Domagala P, Fragola G, Freiberger P, OrtizVigón A, Rousseau P, Tondela J. A prospective non-interventional study to evaluate survival and success of reduced diameter implants made from titanium-zirconium alloy. $\mathrm{J}$ Oral Implantol. 2014 Mar 25.

7. Polack MA, Arzadon JM. Titanium-zirconium implants: Case report. Immediate provisional and restoration using a small-diameter system. Dent Today. 2012 Dec;31(12):64, 66, 68-9.

8. Hasan I, Bourauel C, Mundt T, Stark H, Heinemann F. Biomechanics and load resistance of small-diameter and mini dental implants: a review of literature. Biomed Tech (Berl). 2014 Feb; 59(1):1-5.

9. Gleiznys A, Skirbutis G, Harb A, Barzdziukaite I, Grinyte I. New approach towards mini dental implants and smalldiameter implants: an option for long-term prostheses. Stomatologija. 2012;14(2):39-45.

10. Brandt R, Hollis S, Ahuja S, Adatrow P, Balanoff W. Shortterm objective and subjective evaluation of small-diameter implants used to support and retain mandibular prosthesis. J Tenn Dent Assoc. 2012 Spring;92(1):34-8

11. Patel PB. Maxillary small-diameter implant-retained overdentures. Dent Today. 2013 Jul; 32(7):101-3.

12. Sohrabi K, Mushantat A, Esfandiari S, Feine J. How successful aresmall-diameter implants? A literature review. Clin Oral Implants Res. 2012 May;23(5):515-25. 
13. Ortega-Oller I, Suárez F, Galindo-Moreno P, TorrecillasMartínez L, Monje A, Catena A, Wang HL. The influence of implant diameter on its survival: a meta-analysis based on prospective clinical trials. J Periodontol. 2014 Apr; 85(4):569-80

14. Bidra AS, Almas K. Mini implants for definitive prosthodontic treatment: a systematic review. J Prosthet Dent. 2013 Mar;109(3):156-64.

15. Bourauel C, Aitlahrach M, Heinemann F, Hasan I. Biomechanical finite element analysis of small diameter and short dental implants: extensive study of commercial implants. Biomed Tech (Berl). 2012 Jan 26;57(1):21-32

16. Brian J. Jackson. Small-Diameter Implant Treatment Plan Revision:Management of Complications $\mathrm{J}$ of Oral Implantology . 2016 (3) : 295-298

17. Parfitt, A. M. Bone histomorphometry: standardization of nomenclature, symbols and units. Summary of $\backslash$ proposed system. Bone Miner. 1988; 4(1): p. 1-5.

18. Roberts, W. E., Turley P. K., Brezniak N., et al. Implants: Bone physiology and metabolism. CDA J. 1987; 15(10): p. 54-61

19. Enlow, D. H. A study of the post-natal growth and remodeling of bone. Am J Anat. 1962; 110: p. 79-101.

20. Slaets, E., Naert I., Carmeliet G., et al. Early cortical bone healing around loaded titanium implants: a histological study in the rabbit. Clin Oral Implants Res. 2009; 20(2): p. 126-34.
21. Meredith, N. Assessment of implant stability as a prognostic determinant. Int J Prosthodont. 1998; 11(5): p. 491-501.

22. Friberg, B., Sennerby L., Linden B., et al. Stability measurements of one-stage Branemark implants during healing in mandibles. A clinical resonance frequency analysis study. Int J Oral Maxillofac Surg. 1999; 28(4): p. 266-72.

23. Gedrange, T., Hietschold V., Mai R., et al. An evaluation of resonance frequency analysis for the determination of the primary stability of orthodontic palatal implants. A study in human cadavers. Clin Oral Implants Res. 2005; 16(4): p. 425-31.

24. Abrahamsson, I., Berglundh T., Linder E., et al. Early bone formation adjacent to rough and turned endosseous implant surfaces. An experimental study in the dog. Clin Oral Implants Res. 2004; 15(4): p. 381-92.

25. Lachmann, S., Laval J. Y., Axmann D., et al. Influence of implant geometry on primary insertion stability and simulated peri-implant bone loss: an in vitro study using resonance frequency analysis and damping capacity assessment. Int J Oral Maxillofac Implants. 2011; 26(2): p. 347-55.

26. Turkyilmaz, I., Sennerby L., McGlumphy E. A., et al. Biomechanical aspects of primary implant stability: a human cadaver study. Clin Implant Dent Relat Res. 2009; 11(2): p. 113-9.

27. Quesada-Garcia, M. P., Prados-Sanchez E., Olmedo-Gaya M. V., et al. Measurement of dental implant stability by resonance frequency analysis: a review of the literature. Med Oral Patol Oral Cir Bucal. 2009; 14(10): p. e538-46. 